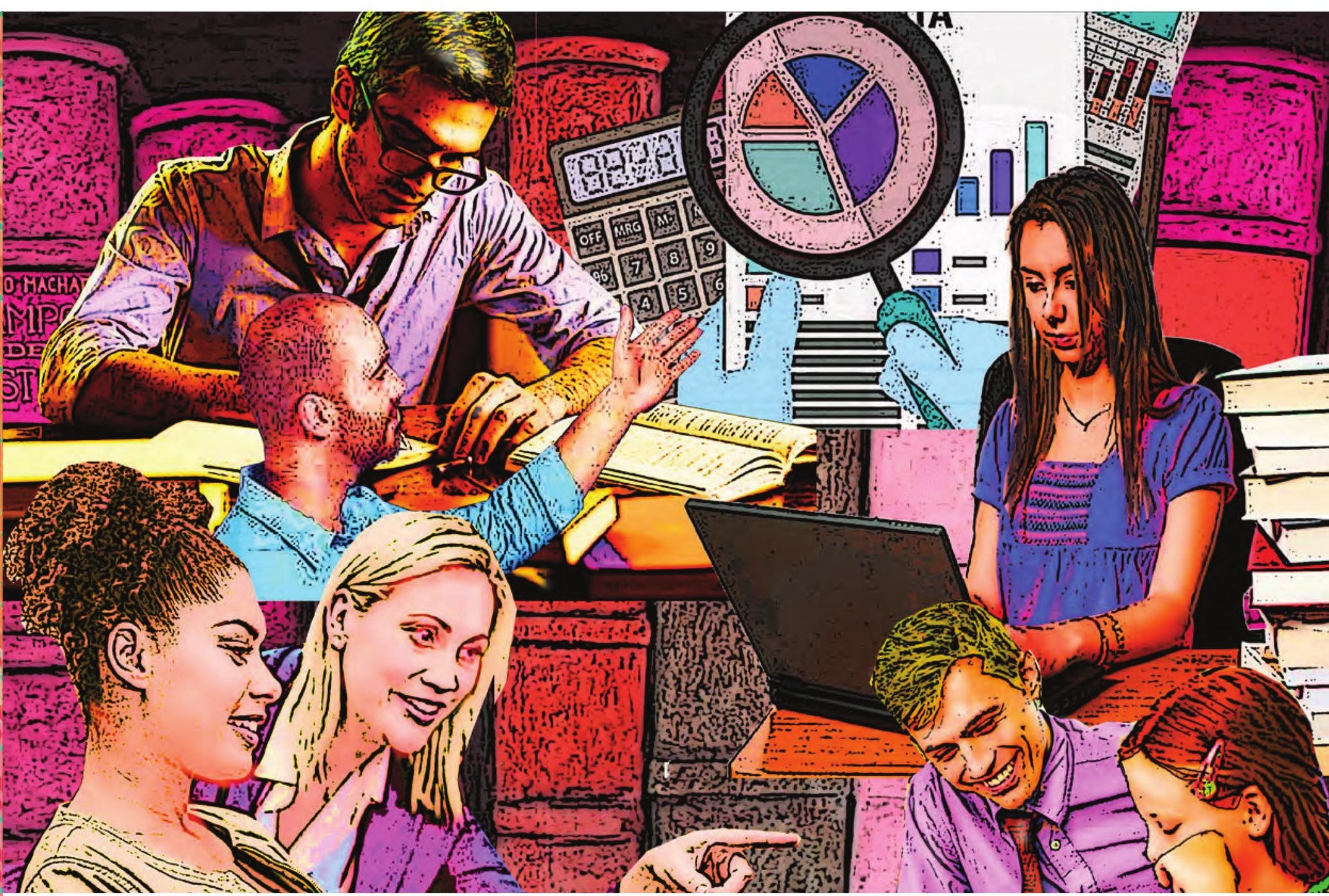

\title{
El profesorado como agente de cambio educativo: entre la docencia y la investigación
}

TEACHERS AS AGENTS OF EDUCATIONAL CHANGE: BETWEEN TEACHING AND RESEARCH PROFESSORES COMO AGENTES DE MUDANÇA EDUCACIONAL: ENTRE ENSINO E PESQUISA Evangelina Cervantes Holguín Pavel Roel Gutiérrez Sandoval 
TEMA: : IMPORTANCIA DE LA INVESTIGACIÓN DE LOS MAESTROS Y MAESTRAS

\section{Evangelina Cervantes Holguín ${ }^{1}$ Pavel Roel Gutiérrez Sandoval²}

Universidad Autónoma de Ciudad Juárez; Doctora en Educación, Universidad de Tijuana; correo electrónico: evangelina.cervantes@uacj.mx

2. Universidad Autónoma de Ciudad Juárez; Doctor en Educación, Universidad de Tijuana; correo electrónico: pavel.gutierrez@uacj.mx

DOI https://doi.org/10.36737/01230425.n38.2020.2316

Fecha de recepción: 3 de febrero de 2020 / Fecha de aprobación: 15 de mayo de 2020

\section{Resumen}

El presente artículo analiza la iniciativa de un grupo de docentes que buscó construir investigación colaborativa, entre una institución de educación superior y diversos centros escolares de educación obligatoria en Ciudad Juárez, Chihuahua, México. El trabajo se basa en la Movilización del Conocimiento y en los principios de la investigación acción. A través del taller investigativo se describe la primera fase, que posibilitó constituir una red de agentes de cambio educativo y un banco de problemáticas que servirán de insumo para realizar proyectos de titulación.

Palabras clave: Formación de docentes, investigación centrada en un problema, investigación participativa, investigación pedagógica, posgrado, tesis.

\section{Abstract}

The purpose of this paper is to analyze the initiative of a group of teachers to build collaborative research between a higher education institution and various compulsory schools in Ciudad Juárez, Chihuahua, Mexico. The work is based on the Knowledge Mobilization and the principles of action research. Through the research workshop, the first phase that enabled the creation of a network of educational change agents and a bank of problems that will serve as input for the realization of degree projects is described.

Keywords: Teacher education, problem focused research, participatory research, educational research, postgraduate education, theses.

\section{Resumo}

O objetivo deste artigo é analisar a iniciativa de um grupo de professores para construir pesquisa colaborativa entre uma instituição de ensino superior e várias escolas obrigatórias em Ciudad Juárez, Chihuahua, México. O trabalho baseia-se na mobilização do conhecimento e nos princípios da pesquisa-ação. Por meio do workshop de pesquisa, é descrita a primeira fase que permitiu a criação de uma rede de agentes de mudança educacional e um banco de problemas que servirão de insumo para a realização de projetos de graduação.

Palavras-chave: Formação de professores, pesquisa focada em um problema, pesquisa participativa, pesquisa pedagógica, pós-graduação, tese. 


\section{Introducción}

$\mathrm{E}$ l presente trabajo analiza la iniciativa de un grupo de docentes, que se encaminó a construir trabajos de investigación colaborativa entre una institución de educación superior y diversos centros escolares de educación obligatoria en Ciudad Juárez, Chihuahua, México. Para hacerlo, se parte de tres supuestos

1. Si la escuela es un "espacio formativo para aprender a enseñar" (Contreras, Rittershaussen, Montecinos, Solís, Núñez y Walker, 2010 , p. 85), también es un lugar para aprender a investigar.

2. La práctica docente puede configurarse como un proceso de investigación donde los maestros se sumergen en "el mundo del aula para comprenderlo de forma crítica y vital, analizando sus mensajes, cuestionando sus propias creencias y planteamientos, contrastando interpretaciones y participando en la reconstrucción permanente de la realidad escolar" (Rodríguez, 2015, p. 92).

3. Los proyectos de investigación generados en el posgrado pueden, en apego a la movilización del conocimiento (KMb), posibilitar el intercambio de saberes entre los productores de investigación (investigadores formales) y los usuarios de los resultados de la investigación (docentes, asesores, directores, supervisores), además de contribuir a reducir la brecha entre investigación y práctica docente (Perines, 2017).

En el contexto mexicano, el arribo al posgrado representa la etapa más alta del Desarrollo Profesional Docente (DPD), que comienza en la formación inicial y se consolida en la superación profesional, entendida como la:

Formación destinada a quienes desean especializarse en campos diversos relativos al quehacer educativo y alcanzar mayores niveles de habilitación profesional y desarrollo [...] a través de estudios de especialización, maestría y doctorado (Secretaría de Educación Pública, 2008, p. 45).

Así, el posgrado en educación tiene la responsabilidad de ofrecer una formación que sobrepase la promoción de "conocimientos teóricos suficientes, pertinentes y actualizados. Supone un replanteamiento curricular (...) que facilite el desarrollo profesional y, con ello, la mejora continua de la práctica” (Mata, Hernández y Centeno, 2019, pp. 83-84), para que los docentes reflexionen sobre qué, cómo y por qué enseñan, lo cual solo se logrará “a través de la vinculación estrecha de su práctica docente con la investigación” (Greybeck, Moreno y Peredo, 1998, párr. 44).

El Centro Chihuahuense de Estudios de Posgrado (CCHEP) es una institución de educación superior, dependiente de la Secretaría de Educación y Deporte del Gobierno del Estado de Chihuahua, encargada de la formación del profesorado de la región a través de dos programas: La Maestría en Desarrollo Educativo, orientada a: "formar profesionales de la educación de alto nivel (...) a través de procesos sistemáticos de investigación en el campo educativo, intervención para la mejora de las organizaciones educativas, así como la evaluación de instituciones, programas y del profesorado" (CCHEP, 2019, párr. 2); y la Maestría en Competencias para la Docencia, dirigida a: "formar profesionales de la educación de alto nivel (...) a través del análisis de las prácticas actuales, detección de problemas de aprendizaje y diseño e implementación de propuestas pedagógicas alternativas" (CCHEP, 2019, párr. 1).

Según el Artículo 24 del reglamento institucional (CCHEP, 2009), para la obtención del grado de maestría los candidatos deberán presentar un informe de investigación, intervención o evaluación. En el camino a la construcción de la tesis, un desafío para los asesores es orientar al estudiantado de los cursos de investigación sobre el ingreso al campo, el nivel de participación en los espacios escolares, la colaboración de los agentes educativos, el estilo de redacción en el informe y la socialización de los resultados con las comunidades escolares. La siguiente experiencia en el aula ilustra dicha conjetura: En una sesión de clase de posgrado, una de las estudiantes comentaba que asistió a una reunión de profesores como estrategia para presentarse ante el colectivo docente y exponer su propuesta de investigación. Como parte del protocolo escolar, el director tomó la palabra; después de dar la bienvenida exclamó:

Durante este ciclo no habrá permiso para hacer investigación. Los profesores que están realizando su maestría deberán buscar otro espacio para hacer su tesis. Luego vienen aquí, nos quitan el tiempo con su lenguaje rebuscado y la escuela, ¿qué gana? ¡Nada!

De lo anterior, el profesorado abrió un espacio para reflexionar sobre las decisiones tomadas por los estudiantes ante el inicio del trabajo de campo, y sobre las dificultades que se presentan al momento de emprender un proyecto de investigación, intervención o evaluación educativas. En el diálogo se palpaba la frustración al no encontrar apertura y apoyo suficiente por parte de las comunidades escolares - pese a que todos los estudiantes son docentes en servicio-; para estos colectivos dichas actividades de indagación y cambio educativo no solo resultaban infructuosas, 
TEMA: : IMPORTANCIA DE LA INVESTIGACIÓN DE LOS MAESTROS Y MAESTRAS

sino que constituían un lastre que se sumaba a las ya numerosas actividades adicionales que pesan sobre la carga docente.

En el esfuerzo por entender la aparente renuencia de los colectivos docentes a desarrollar propuestas de investigación, conviene señalar que el escenario actual ha sido adverso para la escuela pública y para las personas que le dan vida. En México, la última reforma educativa impuso planes, programas y formas de trabajo que situaron al docente como un operario sin capacidad de autogestión; se redujeron los compromisos económicos del Estado con la educación pública y se promovió una campaña denigratoria y de hostigamiento contra el profesorado (Hernández, 2013). A lo anterior se suma la distancia entre la investigación y la práctica educativa, que "avanzan por caminos radicalmente distintos, antagónicos (...) casi irreconciliables” (Perines, 2018b). En opinión de los docentes, la investigación "vive en su preciosa torre de marfil sin que se digne a mirar lo que acontece en las aulas y en las escuelas" (Zorrilla, 2010, p. 81).

En tal contexto, Perines (2018b) encuentra que las principales causas de la crisis de la investigación educativa aluden al profesorado: su escasa formación en investigación; la explicación simplista de la transferencia de los conocimientos a la práctica docente; la desvalorización de sus saberes y la desconfianza que muestra hacia los artículos de investigación. Así, Murillo, Perines y Lomba (2017) afirman que se han generado diversas estrategias -o dispositivos según la propuesta de Souto (2019) - en el devenir de la investigación educativa, para que ésta trastoque la práctica educativa; destacándose la formación inicial y continua del profesorado.

Como contenido curricular en los programas de formación docente, la formación para la investigación usualmente emplea un enfoque con énfasis en la teoría o en la práctica. Al respecto, Korthagen (2010) propone una formación que las una, denominándola "enfoque realista", el cual se caracteriza por trabajar a partir de situaciones reales, promover la reflexión y la intervención guiada y recuperar la teoría -con "t" minúscula- creada por los propios docentes.

Por su parte, Cervantes, Elías y Gutiérrez (2019) proponen acercar la investigación a los docentes, lo cual implica construir "nuevos caminos de relación con las comunidades educativas [que permitan revalorizar] las voces, experiencias y anhelos de las personas involucradas" (p. 4); esos "saberes experienciales" desarrollados por los docentes en el ejercicio de su profesión constituyen el elemento que singulariza su práctica, pues forman un conjunto de "representaciones a partir de las cuales los educadores interpretan, comprenden y orientan su profesión y su práctica cotidiana en todas sus dimensiones" (Tardif, 2004, p. 37).
Considerando que la formación de docentes "juega un rol fundamental para que éstos se involucren activamente en la investigación” (Perines y Campaña, 2019, p. 8), se generó una iniciativa para construir proyectos de investigación colaborativa que permitieran a los estudiantes desarrollar trabajos de titulación pertinentes, en atención a las necesidades del contexto escolar, sean ellas normativas, sentidas, expresadas o comparativas (DizLópez, 2017).

Dado que diversos estudios (Anwaruddin, 2015; Cervantes, 2019; Perines, 2018a; Perines y Murillo, 2017) develan la ausencia de un vínculo real entre las necesidades de los usuarios -docentes, directores, asesores y supervisores- y la producción de conocimiento -investigadores-, la iniciativa se sustentó en los tres rasgos de la propuesta metodológica de Smith (2016): 1) Recuperar el espacio escolar por quienes le dan vida; 2) Implicar a los sujetos educativos en la investigación, de tal manera que transiten de informantes a colaboradores; y 3) Considerar los diversos contextos educativos como espacios para generar nuevos enfoques y modalidades de investigación.

La iniciativa se inspira en el Knowledge Mobilization ( $K M b$ ) o Movilización del Conocimiento, término que alude a la idea de que "el conocimiento debe 'movilizarse', salir, difundirse, generar debate, tener consecuencias y repercusiones en la práctica educativa" (Perines, 2018a, p. 20). Este concepto, acuñado en 2004 por el Consejo de Investigación de Ciencias Sociales y Humanidades de Canadá (SSHRC), se refiere a los "esfuerzos por superar la distancia entre la producción de conocimientos, sus repercusiones prácticas y la política" (Perines, 2017, p. 138); al tiempo, implica los principios de la investigación-acción, participativa, reflexiva y colaborativa, empleada con frecuencia a favor del DPD (Pérez, 2019).

Igualmente, se consideraron dos experiencias internacionales realizadas en Colombia y Uruguay: La Convocatoria Nacional de Extensión Solidaria 2017, impulsada por la Universidad Nacional de Colombia (2017), y el Fondo Sectorial de Educación: investigación de docentes sobre prácticas educativas, creado por la Agencia Nacional de Investigación e Innovación (ANII, 2017); las cuales coinciden en promover la participación de los investigadores universitarios en el diseño e implementación de proyectos con impacto social. En México, las experiencias de este tipo son inéditas. Hoy, con los Programas Nacionales Estratégicos (PRONACES), se invita a los actores sociales a presentar proyectos colaborativos y multidisciplinarios para investigar aquellas problemáticas que, por su importancia y gravedad: "requieren de una atención urgente y de una solución integral" (Gobierno de México, 2019, p. 1). 


\section{Metodología}

Para lograr el objetivo se recurrió al taller investigativo como orientación metodológica. La idea fue abordar desde una perspectiva "integral y participativa, situaciones sociales que requieren algún cambio o desarrollo" (Sandoval, 1996, p. 146), a través de un ejercicio colectivo que permitiera definir un plan específico para recolectar información, analizarla y diseñar acciones de mejora. En atención a la propuesta de Sandoval (1996), el taller investigativo, realizado en las instalaciones del CCHEP, Unidad Juárez, durante el semestre agosto-diciembre de 2019, con una duración aproximada de tres horas, se organizó en cuatro etapas:
1. Encuadre: Se constituyó el taller. En concreto, fueron dos los criterios utilizados para conformar el grupo: ser estudiante o egresado del CCHEP y ser docente, asesor o directivo, en servicio, en algunos de los niveles de la educación básica. En total se convocó a 43 docentes mediante llamada telefónica y la mayoría aceptó; aunque algunos profesores no accedieron, ello se debió principalmente a la incompatibilidad de horarios entre la realización del taller y su trabajo en las escuelas. Como resultado, se conformó un grupo de 22 docentes que se desempeñan como Asesores Técnico-Pedagógicos (ATP), subdirectores, directores y supervisores de educación obligatoria -básica y media superior- en Ciudad Juárez, Chihuahua, México. Los participantes se presentaron e interactuaron con el resto del grupo. Se plantearon los propósitos y se establecieron las metas esperadas.

Tabla 1. Participantes del estudio

\begin{tabular}{|c|c|c|c|c|c|}
\hline Nivel/Función & ATP & Subdirectores & Directores & Supervisores & Total \\
\hline Preescolar & 1 & & 1 & 1 & 3 \\
\hline Primaria & 3 & 1 & 8 & 1 & 13 \\
\hline Secundaria & & & 2 & & 2 \\
\hline Media superior & & & 1 & & 1 \\
\hline Educación física & & & & 2 & 2 \\
\hline Educación especial & & & 1 & & 1 \\
\hline Total & 4 & 1 & 13 & 4 & 22 \\
\hline
\end{tabular}

Nota. Fuente: Elaboración propia, con base en las características de los docentes participantes del estudio

2. Diagnóstico: Se realizó un ejercicio inicial de detección de necesidades, con el propósito de recuperar las experiencias y preocupaciones de los participantes en torno a aquellos problemas observados respecto a estudiantes, docentes y familia, tanto dentro como fuera de la escuela. Las preguntas generales planteadas en el taller fueron establecidas previamente, sin embargo, se consideró dar tiempo para atender los interrogantes que surgirían a partir del diálogo entre docentes. La conversación se orientó considerando algunas cuestiones, por ejemplo: ¿Qué situaciones dentro y fuera de la escuela pueden incentivar trabajos de investigación, intervención o evaluación?; ¿¿cómo pueden acercarse a ellas los docentes que estudian una maestría en la institución?; ¿quiénes pueden apoyar estas iniciativas?; ¿por dónde empezar?

3. Identificación-valoración y formulación: Se identificaron, valoraron y formularon las líneas de acción en función de los resultados del diagnóstico. Se examinaron las alternativas de acción y se decidió constituir un grupo de trabajo, el cual se autodenominó "Red de agentes de cambio educativo", planteando el objetivo de: Fomentar la participación de los colectivos escolares y las comunidades educativas en el 
diseño e implementación de proyectos de titulación en las modalidades de investigación, intervención y evaluación; para generar acciones colectivas de mejora a problemas planteados por docentes, directivos, asesores y supervisores escolares. Esta acción constituyó el punto de partida y el mayor desafío, pues entre las instituciones de educación superior y escuelas de educación obligatoria hay: "demasiada distancia, no existen instancias explícitas de diálogo (...) [Y] La superación de esta problemática requiere que (...) se definan temáticas de interés de los profesores y acciones concretas para acercar la investigación a la práctica docente" (Perines y Murillo, 2017, p. 102)

4. 4.Estructuración y concertación: Se estructuró un plan de trabajo para implementar las líneas de acción identificadas, entonces se establecieron diversos compromisos organizados en tres etapas:

a. Etapa 1. Inscripción de situaciones en el "Banco de problemas educativos". Los colectivos escolares y las comunidades educativas de cualquier parte de Ciudad Juárez, docentes, estudiantes y egresados del CCHEP, podrán inscribir un problema de interés en el banco de problemas educativos; dichos problemas serán tomados como referente para diseñar proyectos de titulación en las modalidades de investigación, intervención y evaluación.

b. Etapa 2. Presentación de problemas educativos. Los problemas serán revisados por los directores de tesis y sus equipos de trabajo, y seleccionarán algunos para diseñar su proyecto de titulación. Los avances de los diferentes proyectos se presentarán semestralmente en el Coloquio de Investigación o en el Encuentro de Intervención.

c. Etapa 3. Encuentro con las escuelas y comunidades educativas. Cada director/a de tesis, y sus estudiantes, trabajará colaborativamente con los responsables de la propuesta, quienes tendrán la responsabilidad de otorgar las facilidades para la realización del trabajo de campo.

En correspondencia con los propósitos del presente texto, se examinan los resultados del taller investigativo que representó el primer paso de la iniciativa. El análisis de los datos se realizó con base en algunos de los principios de la Teoría Fundamentada (Glaser y Strauss, 2017), lo cual implicó una lectura reflexiva para identificar categorías relevantes y crear códigos que, a su vez, fueron analizados y comparados entre sí (Gibbs, 2014).
Para la codificación y la jerarquización de los datos empíricos se emplearon tablas cualitativas por su conveniencia para "mostrar texto procedente de todos los puntos del conjunto de datos" (Gibbs, 2014, p. 109).

\section{Resultados}

Como guía para el lector, los resultados se presentarán a partir de las cuatro etapas trabajadas en el taller investigativo.

\section{La invitación al taller: De vuelta al salón de clases}

Un estudio realizado en Argentina concluyó que los educadores de todos los subsistemas tienen "pocas oportunidades de formarse para la investigación y, también, de implementarla" (ANII, 2019, p. 9), situación que se puede extender al contexto mexicano, donde son escasas las oportunidades ofrecidas al profesorado de educación obligatoria para formarse como investigador educativo (Cervantes, 2019); pero: ¿Por qué formar en investigación a los docentes? Desde la perspectiva de Catalán (2017) es necesario formar profesores para que "no sean exclusivamente consumidores de conocimiento, sino que también contribuyan a producirlo" ( $p$. 28).

Así, partiendo de la idea de que los líderes escolares pueden impactar en el DPD de sus colectivos (Day, 2005; Muñóz, 2019), en especial al "contribuir a la formación de los docentes (...), obtener recursos adicionales para apoyar el aprendizaje de los estudiantes [y] proveer liderazgo pedagógico" (Montecinos, Walker, Cortez y Maldonado, 2015, p. 23), se extendió la invitación a los egresados del CCHEP que, a la fecha, ocupan algún cargo directivo en los diversos niveles de la educación obligatoria, pensando en el hecho de que, como afirmaron Perines y Murillo (2017), los equipos directivos pueden ser líderes en "la creación de una cultura de aprendizaje donde la investigación sea un recurso útil para mejorar" (p. 103).

En este sentido, la invitación a los egresados del CCHEP para participar en el taller, se presentó como una oportunidad para apoyar a los estudiantes en la realización de trabajos de titulación pertinentes y en la posibilidad de colaborar con sus escuelas, colegas, estudiantes o padres de familia, al atender una problemática real. Esta acción coincidió con una de las características del modelo educativo institucional, en donde se asume la formación docente desde múltiples niveles y con diversos actores, materializándose en una concepción de la formación como un ejercicio colectivo al interior de las escuelas. Así, se une con la idea de Contreras, et al. (2010), en el sentido de que se trata de generar un: "Proceso 
colaborativo empoderante que facilite la discusión acerca de los problemas y necesidades (...) de los profesores, la validación de experiencias como base para una reflexión crítica y la proyección de mejoras a partir de los resultados" (p. 87).

\section{El taller: El encuentro con los otros}

El taller se pensó como un espacio colectivo de intercambio de ideas, una suma de esfuerzos y metas comunes que permitiera el encuentro entre investigadores -directores de tesis- y docentes con funciones de dirección, asesoría y supervisión escolar. En concreto, se constituyó como un lugar de reconciliación entre los investigadores y los agentes educativos, en especial, porque: "Tienen diferentes expectativas de la investigación: quienes trabajan en las escuelas buscan nuevas soluciones a los problemas concretos que los aquejan, mientras que los investigadores buscan generar y publicar nuevos conocimientos" (Perines, 2018b, p. 11).
Para el diseño de proyectos de investigación impulsados y desarrollados por los propios docentes, se invitó a los participantes a identificar las necesidades de sus comunidades, buscando desarrollar una "investigación educativa propia, que recoja las problemáticas de sus escuelas, identifique sus propias estrategias para la mejora y promueva el desarrollo de políticas educativas contextualizadas a la realidad de sus aulas" (Murillo y Martínez, 2019 , p. 24). En el mismo sentido, se priorizó la voz de los docentes para distinguir las problemáticas iniciales, lo que implicó pasar a investigar "con", en vez de "investigar a" (Fernández, 2001, p. 166); es decir, transitando desde "ser investigadores de una realidad, a ser investigadores en y con los agentes de dicha realidad" (Perines y Murillo, 2017, p. 101). A continuación, la Tabla 2 permite observar las problemáticas identificadas.

Tabla 2. Necesidades educativas por sujeto y nivel educativo

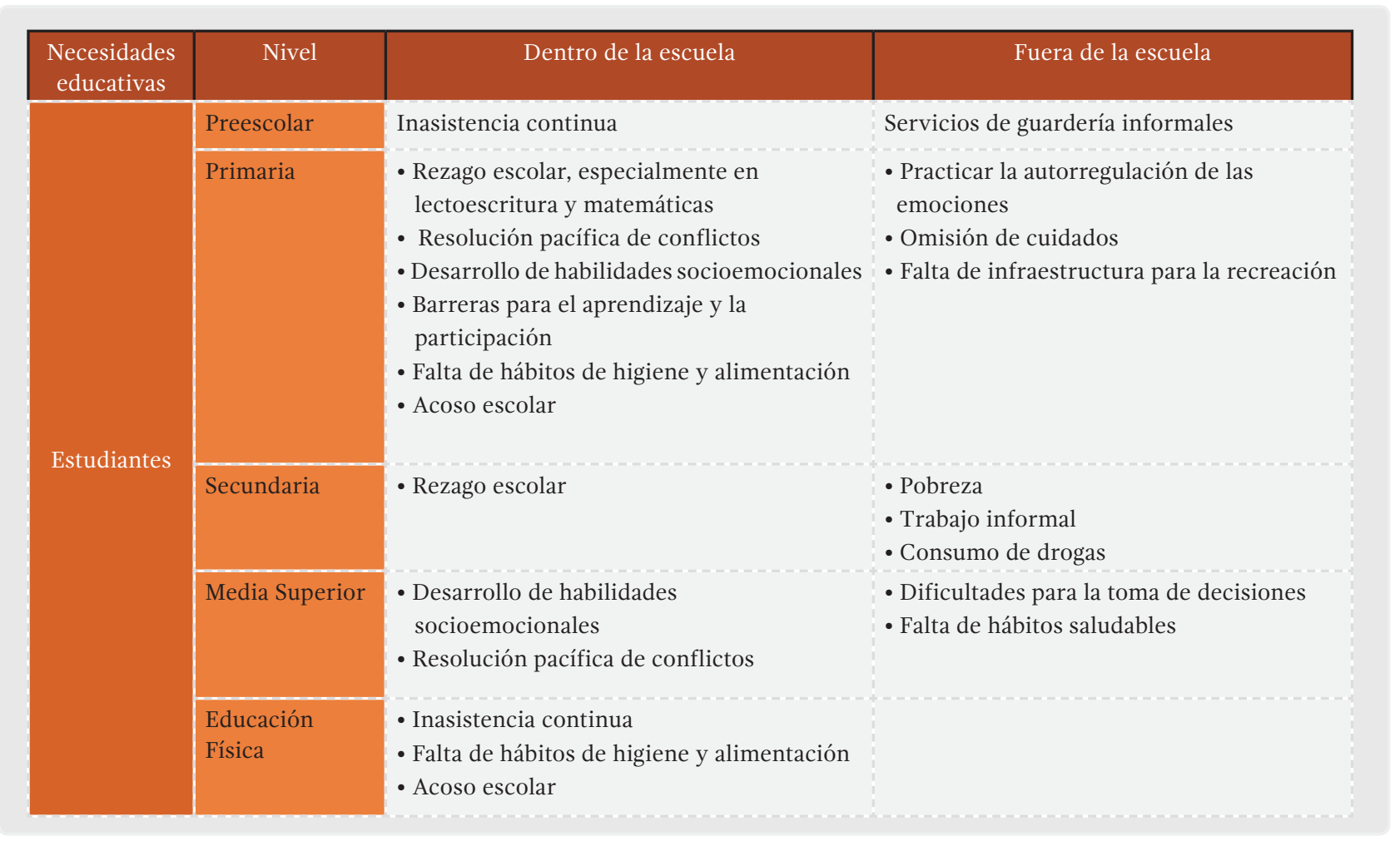

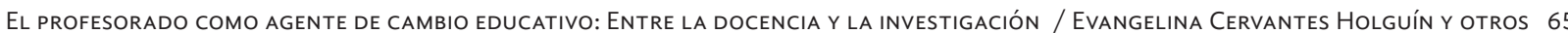


TEMA: : IMPORTANCIA DE LA INVESTIGACIÓN DE LOS MAESTROS Y MAESTRAS

\begin{tabular}{|c|c|c|c|}
\hline $\begin{array}{l}\text { Necesidades } \\
\text { educativas }\end{array}$ & Nivel & Dentro de la escuela & Fuera de la escuela \\
\hline \multirow{5}{*}{ Docentes } & Preescolar & $\begin{array}{l}\text { - Atención a la diversidad } \\
\text { - Seguimiento de proyectos escolares } \\
\text { - Actitudes negativas ante la práctica } \\
\text { docente }\end{array}$ & $\begin{array}{l}\text { - Insuficientes espacios de formación } \\
\text { docente }\end{array}$ \\
\hline & Primaria & $\begin{array}{l}\text { - Desarrollo de habilidades } \\
\text { socioemocionales } \\
\text { - Apatía ante la formación continua } \\
\text { - Profesorado en edad de jubilación } \\
\text { - Falta de competencias para atender la } \\
\text { diversidad } \\
\text { - Sobrecarga administrativa } \\
\text { - Carencia de habilidades de comunicación } \\
\text { efectiva } \\
\text { - Falta de planeación didáctica } \\
\text { - Uso ineficiente del tiempo } \\
\text { - Ausencia del trabajo colaborativo }\end{array}$ & $\begin{array}{l}\text { - Alcoholismo } \\
\text { - Violencia intrafamiliar } \\
\text { - Burnout }\end{array}$ \\
\hline & Secundaria & $\begin{array}{l}\text { - Falta de actualización } \\
\text { - Dependencia de la dirección escolar }\end{array}$ & - Movilidad docente \\
\hline & Media Superior & $\begin{array}{l}\text { - Visión desvinculada de la función social } \\
\text { de la escuela }\end{array}$ & $\begin{array}{l}\text { - Insuficientes competencias para innovar } \\
\text { la práctica docente }\end{array}$ \\
\hline & $\begin{array}{l}\text { Educación } \\
\text { Física }\end{array}$ & $\begin{array}{l}\text { - Desconocimiento de los beneficios de la } \\
\text { Educación Física } \\
\text { - Uso ineficiente del tiempo } \\
\text { - Falta de espacios y materiales }\end{array}$ & - Falta de rendición de cuentas \\
\hline \multirow[b]{2}{*}{ Familia } & Preescolar & $\begin{array}{l}\text { - Mínima participación en las actividades } \\
\text { escolares }\end{array}$ & - Omisión de cuidados \\
\hline & Primaria & $\begin{array}{l}\text { - Mínima participación en las actividades } \\
\text { escolares } \\
\text { - Desvalorización de la función docente } \\
\text { - Población flotante }\end{array}$ & $\begin{array}{l}\text { - Divorcios contenciosos } \\
\text { - Pobreza } \\
\text {-Participación en la delincuencia } \\
\text { organizada } \\
\text {-Desconocimiento de instancias externas } \\
\text { de apoyo a la niñez } \\
\text { - Migración } \\
\text { - Inestabilidad laboral } \\
\text { - Falta de hábitos de higiene y alimentación }\end{array}$ \\
\hline
\end{tabular}




\begin{tabular}{|c|l|l|l|}
\hline $\begin{array}{c}\text { Necesidades } \\
\text { educativas }\end{array}$ & \multicolumn{1}{|c|}{ Nivel } & \multicolumn{1}{|c|}{ Dentro de la escuela } & \multicolumn{1}{c|}{ Fuera de la escuela } \\
\hline \multirow{2}{*}{ Familia } & Secundaria & - Distancia entre docentes y familias & $\begin{array}{l}\text { Extensa jornada laboral } \\
\text { - Lengua }\end{array}$ \\
\cline { 2 - 5 } & Media Superior & $\begin{array}{l}\text { Excesiva preocupación por las notas de } \\
\text { los estudiantes }\end{array}$ & $\begin{array}{l}\text { Carencia de habilidades de comunicación } \\
\text { efectiva }\end{array}$ \\
\cline { 2 - 5 } & $\begin{array}{l}\text { Educación } \\
\text { Física }\end{array}$ & $\begin{array}{l}\text { Mínima participación en las actividades } \\
\text { escolares }\end{array}$ & $\begin{array}{l}\text { Desconocimiento de los beneficios de la } \\
\text { Educación Física }\end{array}$ \\
\hline
\end{tabular}

Nota. Fuente: Elaboración propia con base en el material primario

Las problemáticas enunciadas por los participantes destacan los cada vez mayores desafíos de la docencia, en medio de momentos de un "alto nivel de ambigüedad, incertidumbre y complejidad" (Peña y Pérez, 2019, p. 570), que hacen indispensable la investigación para trabajar los "múltiples retos de las pedagogías emergentes, como la inclusiva, ambiental, de género, intercultural (...), así como las necesidades de aprendizaje de sus alumnos y los problemas o situaciones existentes en el contexto" (Calixto, 2019, p. 22).

\section{Conformemos una red: De docentes-investigadores a agentes de cambio educativo}

A principios del siglo XXI, Torres (2000) señalaba que: "para cambiar la educación es preciso trabajar con los docentes, no contra ellos o a sus espaldas, asumiéndolos (...) como aliados y como sujetos del cambio" (p. 1). En este sentido, un primer logro del trabajo fue la conformación de una red que, en tanto comunidad de aprendizaje, buscaba la: "combinación del saber educativo (experiencia práctica) con el saber sobre la educación (conocimientos adquiridos mediante la investigación)" (Day, 2005, p. 67).

Con esta propuesta de formación situada se buscaba descolonizar el conocimiento para servir, con mayores elementos, a los docentes y sus comunidades, a través de la realización de proyectos de investigación que tendieran a la producción -horizontal y simétrica- de conocimiento, como condición para crear un mundo socialmente justo. En ella, los docentes desarrollan su capacidad de agencia para "construir saberes (...) superar la pasividad y la condición de espectadores y reproductores” (Martínez, 2005, p. 6).

\section{El banco de problemas: De la tesis al cambio educativo}

Desde la formación práctica (Muñoz, 2019) se generó un modelo de instrucción para la investigación, basado en problemas y con una lógica de "abajo hacia arriba" donde, a partir de la identificación de problemas escolares concretos, se diseñaron trayectos formativos vinculados a la construcción del informe para la obtención del grado de maestría. Se trata de una modalidad de investigación formativa que moviliza "un conjunto de estrategias de aprendizaje de la indagación, la exploración de la realidad y la construcción de conocimiento" (Pirela, Pulido y Mancipe, 2015, p. 51).

Este momento del proceso alude al poder transformador de la investigación educativa cuando responde a las necesidades contextuales de los docentes (Muñoz y Garay, 2015). En tanto que cuando los maestros "investigan los fenómenos que acontecen en sus propias aulas, se vuelven más críticos y reflexivos, generan conocimiento práctico y útil que potencializa la mejora de su práctica docente y se renuevan continuamente como profesores" (Mendoza y Roux, 2015, p. 49).

Durante el trascurso de esta etapa se determinó la corresponsabilidad como un elemento clave en el éxito del proyecto, pues los resultados positivos se amplían cuando "ambas instituciones asumen responsabilidades, autoridad y rendición de cuentas compartidas" (Montecinos, et al., 2015, p. 26). La definición de compromisos se estableció en un "contrato de investigación entre todos los agentes” (Fernández, 2001, p. 166), para evitar situaciones de carácter logístico, organizativo, ético y deontológico que perjudicaran el trabajo colaborativo.

Mientras los directivos escolares aceptaron otorgar facilidades para realizar los proyectos, los estudiantes y sus asesores de tesis 
TEMA: : IMPORTANCIA DE LA INVESTIGACIÓN DE LOS MAESTROS Y MAESTRAS

-como equipos de investigación-, se comprometieron a trabajar con las comunidades bajo estudio y presentarles los hallazgos una vez se terminara. Aunque la "devolución” de resultados (Zarco, Ramasco, Pedraz y Palmar, 2019) es un requisito formal en la investigación, aquí se propone como una herramienta para provocar el diálogo con la institución, lo que implica una "mayor interacción y reflexión con los directivos y con los profesores, además de explicaciones más precisas de los resultados obtenidos” (Perines y Murillo, 2017, p. 101).

\section{Conclusiones}

El texto presentado analizó la experiencia de un grupo de docentes en torno a una iniciativa institucional que buscó generar proyectos de investigación colaborativa para disminuir la separación entre la investigación y la práctica docente. Así, considerando la "inexistencia aún de una masa crítica de docentes que cuenten con formación específica y oportunidades para realizar investigación educativa" (ANII, 2019, p. 9), se buscó generar un puente entre la necesidad de los estudiantes de realizar investigación, usualmente motivada por la culminación de la tesis y la obtención del grado académico (Fernández y Wainerman, 2015), y los crecientes desafíos que enfrentan los colectivos escolares.

Aunque la descripción solo da cuenta de la primera fase del proyecto, permite analizar los retos al innovar los procesos de titulación en el posgrado -comúnmente centrados en la burocracia escolar y los criterios de eficiencia terminal-. La propuesta es transitar, desde el mero desarrollo de competencias investigativas, hacia la promoción de estrategias de formación que "hagan de la investigación un estilo de vida" (Fontaines, Carhuachín, Zenteno y Tusa, 2018, p. 112).

Dado que la investigación educativa constituye un "entramado de prácticas sociales que se desarrolla en función de contextos históricos específicos y marcos institucionales que lo condicionan y le otorgan orientaciones particulares" (Palamidessi, Gorostiaga y Suasnábar, 2014, p. 50), se propone avanzar en la implementación de trayectos formativos plurales, contextuales y emancipadores, que contribuyan a la justicia social o simplemente a vías "menos injustas" (Aguilar, 2017, p. 1).

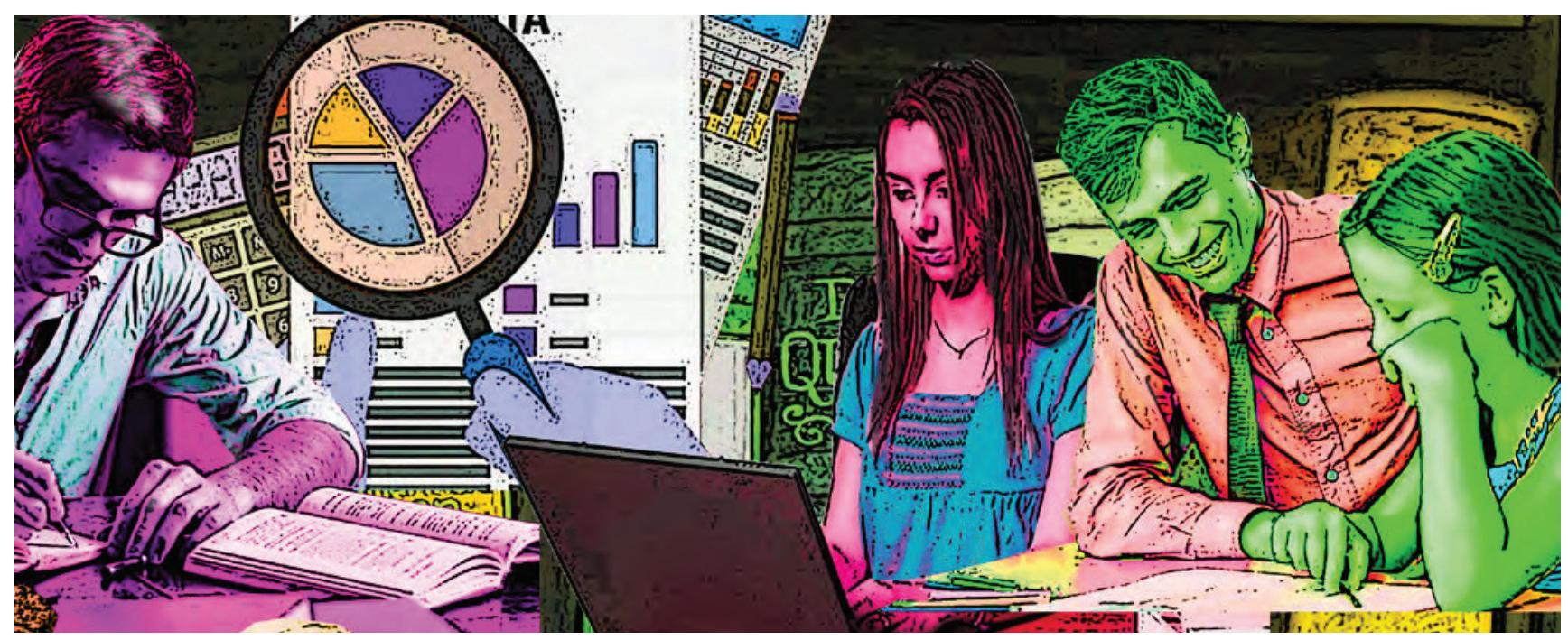




\section{Referencias}

Agencia Nacional de Investigación e Innovación (ANII). (2017). Investigación desde la perspectiva de los educadores sobre sus prácticas educativas. Obtenido desde https://www.anii.org.uy/apoyos/investigacion/78/fondo-sectorial-deeducacion-investigacion-de-docentes-sobre-practicas-educativas/

Agencia Nacional de Investigación e Innovación (ANII). (2019). Fondo Sectorial de Educación «Investigación desde la perspectiva de los educadores sobre sus prácticas educativas». Convocatoria 2015. Montevideo: ANII.

Aguilar, J. (2017). Justicia educativa: itinerario de su historia conceptual en México. Revista electrónica de investigación educativa, 19(2), pp. 1-11. DOI: http://dx.doi.org/10.24320/redie.2017.19.2.1216

Anwaruddin, S. M. (2015). Teachers' engagement with educational research: Toward a conceptual framework for locallybased interpretive communities. Education Policy Analysis Archives, 23(40), pp. 1-25. DOI: http://dx.doi.org/10.14507/ epaa.v23.1776

Banegas, D. L. (2018). Towards understanding EFL teachers' conceptions of research: Findings from Argentina. Profile: Issues in Teachers' Professional Development, 20(1), pp. 57-72. DOI: http://dx.doi.org/10.15446/profile.v20n1.61881

Calixto, R. (2019). Estudio exploratorio de las competencias investigativas de los futuros docentes de Educación Primaria. Magister, 31(1), pp. 17-23. DOI: https://doi.org/10.17811/msg.31.1.2019.17-24

Catalán, J. (2017). Investigación y docencia: una vinculación necesaria y posible. En Araya, F. Desarrollo del pensamiento geográfico. Aportes para la vinculación entre investigación y docencia (pp. 17-38). La Serena: Universidad de la Serena.

Centro Chihuahuense de Estudios de Posgrado (CCHEP). (2009). Reglamento de la institución. Chihuahua: CCHEP.

Centro Chihuahuense de Estudios de Posgrado (CCHEP). (2019). Oferta académica. Obtenido desde https://cchepjrz.wixsite. com/cchepjuarez/oferta-academica

Cervantes, E. (2019). Un acercamiento a la formación de docentes como investigadores educativos en México. REICE. Revista Iberoamericana sobre Calidad, Eficacia y Cambio en Educación, 17(4), pp. 59-74. DOI: https://doi.org/10.15366/ reice2019.17.4.003

Cervantes, E., Elías, J. A., y Gutiérrez, P. R. (2019). Hacia una formación de investigadores educativos con actitud decolonizadora. XV Congreso Nacional de Investigación Educativa. Acapulco: Consejo Mexicano de Investigación Educativa (COMIE), pp. 1-9. Obtenido desde http://www.comie.org.mx/congreso/memoriaelectronica/v15/seccion4.htm

Contreras, I., Rittershaussen, S., Montecinos, C., Solís, M. C., Núñez, C., y Walker, H. (2010). La escuela como espacio para aprender a enseñar: Visiones desde los programas de formación de profesores de educación media. Estudios Pedagógicos, XXXVI(1), pp. 85-105. DOI: 10.4067/S0718-07052010000100004

Day, C. (2005). Formar docentes. Cómo, cuándo y en qué condiciones aprende el profesorado. Madrid: Narcea.

Diz-López, M. J. (2017). Necesidades formativas. Modelos para su análisis y evaluación. Revista de estudios e investigación en psicología y educación, No 6, pp. 6-10. DOI: https://doi.org/10.17979/reipe.2017.0.06.2132

Fernández, A. (2001). Valoración del impacto de la investigación educativa sobre la práctica docente. Revista de Educación, No. 324, pp. 155-170. DOI: 10.4438/1988-592X-0034-8082-RE

Fernández, L., y Wainerman, C. (2015). La dirección de tesis de doctorado: ¿Una práctica pedagógica? Perfiles Educativos, XXXVII(148), pp. 156-171. Obtenido desde https://www.iisue.unam.mx/perfiles/descargas/pdf/2015-148-156-171 
TEMA: : IMPORTANCIA DE LA INVESTIGACIÓN DE LOS MAESTROS Y MAESTRAS

Fontaines, T., Carhuachín, A. I., Zenteno, F. A., y Tusa, F. (2018). Competencias formativas de los investigadores noveles según los investigadores consolidados. Educación, XXVII(53), pp. 107-127. DOI: https://doi.org/10.18800/ educacion.201802.007

Gibbs, G. (2014). El análisis de datos cualitativos en investigación cualitativa. Madrid: Morata.

Glaser, B. G., y Strauss, A. L. (2017). The discovery of grounded theory: strategies for qualitative research. New York: Routledge. DOI: https://doi.org/10.4324/9780203793206

Gobierno de México. (2019). Programas nacionales estratégicos del CONACYT (PRONACES). Obtenido desde https://www. gob.mx/cms/uploads/attachment/file/448752/Programas_Nacionales_Estrat_gicos.pdf

Greybeck, B., Moreno, M. G., y Peredo, M. A. (1998). Reflexiones acerca de la formación de docentes. Educar, No. 5, pp. 1522. Obtenido desde http://www.quadernsdigitals.net/datos_web/articles/educar/numero5/reflexiones.htm

Hernández, L. (2013). No habrá recreo (Contra-reforma constitucional y desobediencia magisterial). México: Rosa Luxemburg Stiftung y Para leer en libertad.

Korthagen, F. A. (2010). La práctica, la teoría y la persona en la formación del profesorado. Revista Interuniversitaria de Formación del Profesorado, 68(24), pp. 83-101. Obtenido desde https://core.ac.uk/download/pdf/41577213.pdf

Martínez, M. C. (2005). La figura del maestro como sujeto político. El lugar de los colectivos y redes pedagógicas en su agenciamiento. Nodos y nudos, 2(19), pp. 4-12. DOI: https://doi.org/10.17227/01224328.1263

Mata, A. M., Hernández, P., y Centeno, G. E. (2019). Proceso de aprendizaje reflexivo en la investigación para la docencia. Revista Panamericana de Pedagogía. Saberes y quehaceres del pedagogo, No. 28, pp. 69-86. Obtenido desde http:// portalderevistasdelaup.mx/revistapedagogia/index.php/pedagogia/article/download/323/330

Mendoza, J. L., y Roux, R. (2015). La investigación docente y el desarrollo profesional continuo: Un estudio de caso en el noreste mexicano. Innovación Educativa, 16(70), pp. 43-60. Obtenido desde http://www.scielo.org.mx/scielo. php?script=sci_arttext\&pid=S1665-26732016000100043\&lng=es\&tlng=es

Montecinos, C., Walker, H., Cortez, M., y Maldonado, F. (2015). ¿Por qué y para qué los centros escolares aceptan ser un sitio de práctica para las carreras de pedagogía? Perspectivas de docentes directivos. Páginas de Educación, 6(1), pp. 1-32. DOI: https://doi.org/10.22235/pe.v6i1.526

Muñóz, G. (2019). Aprendizaje para una política de formación de directores y directoras en Chile. Seminario Formación de Líderes del Sistema Escolar (1a Parte). Santiago: Líderes educativos. Obtenido desde https://www.youtube.com/ watch?time_continue $=23 \& \mathrm{v}=\mathrm{RMD} 0 \mathrm{dNk}-\mathrm{i} 0 \mathrm{w} \&$ feature $=$ emb_logo

Muñóz, M., y Garay, F. (2015). La investigación como forma de desarrollo profesional docente: Retos y perspectivas. Estudios Pedagógicos, XLI(2), pp. 389-399. DOI: http://dx.doi.org/10.4067/S0718-07052015000200023

Murillo, F. J., y Martínez, C. (2019). Una mirada a la investigación educativa en América Latina a partir de sus artículos. REICE Revista Iberoamericana sobre Calidad, Eficacia y Cambio en Educación, 17(2), pp. 5-25. DOI: https://doi.org/10.15366/ reice2019.17.2.001

Murillo, F. J., Perines, H. A., y Lomba, L. (2017). La comunicación de la investigación educativa. Una aproximación a la relación entre la investigación, su difusión y la práctica docente. Profesorado. Revista de currículum y formación del profesorado, 21(3), pp. 183-200. Obtenido desde https://recyt.fecyt.es/index.php/profesorado/article/view/59794/38527

Palamidessi, M., Gorostiaga, J. M., y Suasnábar, C. (2014). El desarrollo de la investigación educativa. Perfiles Educativos, XXXVI(143), pp. 49-66. DOI: https://doi.org/10.22201/iisue.24486167e.2014.143.44022

Peña, N., y Pérez, Á. I. (2019). Las disposiciones subjetivas de los docentes en la superación de las resistencias al cambio ante procesos cíclicos de formación basados en la investigación (Lesson Study): Estudio de un caso. Revista Complutense de Educación, 30(2), pp. 569-587. DOI: http://dx.doi.org/10.5209/RCED.57780

Pérez, M. (2019). La investigación acción en la práctica docente. Un análisis bibliométrico (2003-2017). MAGIS. Revista Internacional de Investigación en Educación, 12(24), pp. 177-192. DOI: 10.11144/Javeriana.m10-20.ncev

Perines, H. (2017). Movilización del conocimiento en educación. Conexión entre la investigación, la política y la práctica: una aproximación teórica. Páginas de Educación, 10(1), pp. 137-150. DOI: http://dx.doi.org/10.22235/pe.v10i1.1362 
Perines, H. (2018a). Cómo conseguir que la investigación educativa impacte en la práctica de los profesores. Estudios sobre Educación, No. 34, pp. 9-27. DOI: http://dx.doi.org/10.15581/004.34.9-27

Perines, H. (2018b). ¿̨Por qué la investigación educativa no impacta en la práctica docente? Estudios sobre educación, No. 34, pp. 9-27. DOI: 10.15581/004.34.9-27

Perines, H., y Campaña, K. (2019). La alfabetización de los futuros docentes en investigación educativa: Una reflexión teórica desde el contexto de Chile. Revista Caribeña de Investigación Educativa (RECIE), 3(1), pp. 7-18. DOI: https:// doi.org/10.32541/recie.2019.v3i1.pp7-18

Perines, H., y Murillo, F. J. (2017). ¿Cómo mejorar la investigación educativa? Sugerencias de los docentes. Revista de la Educación Superior, 46(181), pp. 89-104. DOI: http://dx.doi.org/10.1016/j.resu.2016.11.003

Pirela, J., Pulido, N. J., y Mancipe, E. (2015). Componentes y dimensiones de la investigación formativa en ciencias de la información. Enl@ce: Revista venezolana de información, tecnología y conocimiento, 12(3), pp. 48-70. Obtenido desde https://produccioncientificaluz.org/index.php/enlace/article/view/20627

Rodríguez, J. M. (2015). Cambios educativos asociados a las prácticas de enseñanza del docente. Magister, pp. 91-96. DOI: http://dx.doi.org/10.1016/j.magis.2015.12.004

Sandoval, C. A. (1996). Investigación cualitativa. Bogotá: Instituto Colombiano para el Fomento de la Educación Superior (ICFES).

Secretaría de Educación Pública (SEP). (2008). Sistema Nacional de Formación Continua y Superación Profesional de Maestros en Servicio. México: Subsecretaría de Educación Básica. Obtenido desde http://wbgfiles. worldbank.org/documents/hdn/ed/saber/supporting_doc/LCR/Teachers/Mexico/AS_S6_MEXICO_ ContinuousProfesionalDevelopmentSystem_07.pdf

Smith, L. T. (2016). A decolonizar las metodologías. Investigación y pueblos indígenas. Santiago: LOM Ediciones.

Souto, M. (2019). Acerca de la noción de dispositivo en la formación universitaria. Educación, Lenguaje y Sociedad, XVI(16), pp. 1-16. DOI: http://dx.doi.org/10.19137/els-2019-161602

Tardif, M. (2004). Los saberes del docente y su desarrollo profesional. Madrid: Narcea.

Torres, R. M. (2000). De agentes de la reforma a sujetos del cambio: La encrucijada docente en América Latina. Perspectivas, XXX(2), pp. 1-21. Obtenido desde https://www.oei.es/historico/docentes/articulos/agentes_de_reforma_sujetos_ cambio_torres.pdf

Universidad Nacional de Colombia. (2017). Convocatoria Nacional de Extensión Solidaria 2017: Ciencia, tecnología e innovación para la construcción de tejido social. Obtenido desde http://investigacion.unal.edu.co/boletin/notas-boletin-uninvestiga/news/la-universidad-nacional-de-colombia-escucha-los-problemas-de-las-comunidades/

Zarco, J., Ramasco, M., Pedraz, A., y Palmar, A. M. (2019). Investigación cualitativa en salud. Madrid: Centro de Investigaciones Sociales.

Zorrilla, M. (2010). Investigación educativa, políticas públicas y práctica docente. Triángulo de geometría desconocida. REICE. Revista Iberoamericana sobre calidad, eficacia y cambio en educación, 8(2), pp. 74-92. Obtenido desde http:// www.rinace.net/reice/numeros/arts/vol8num2/art4.pdf 